BNL -67691

\title{
VARIABLE MOMENTUM COMPACTION LATTICE STUDIES
}

\author{
S. Kramer and J.B. Murphy \\ National Synchrotron Light Source \\ Brookhaven National Laboratory \\ Upton, New York 11973-5000, USA
}

March 1999

\author{
National Synchrotron Light Source \\ Brookhaven National Laboratory \\ Operated by \\ Brookhaven Science Associates \\ Upton, NY 11973
}

Under Contract with the United States Department of Energy

Contract Number DE-AC02-98CH10886 


\section{DISCLAIMER}

This report was prepared as an account of work sponsored by an agency of the United States Government. Neither the United States Government nor any agency thereof, nor any of their employees, nor any of their contractors, subcontractors or their employees, makes any warranty, express or implied, or assumes any legal liability or responsibility for the accuracy, completeness, or any third party's use or the results of such use of any information, apparatus, product, or process disclosed, or represents that its use would not infringe privately owned rights. Reference herein to any specific commercial product, process, or service by trade name, trademark, manufacturer, or otherwise, does not necessary constitute or imply its endorsement, recommendation, or favoring by the United States Government or any agency thereof or its contractors or subcontractors. The views and opinions of authors expresses herein do not necessarily state to reflect those of the United States Government or any agency thereof. 


\title{
Variable Momentum Compaction Lattice Studies*
}

\author{
$\underline{\text { S.L. Kramer }}^{\#}$ and J.B. Murphy, BNL, Upton, NY 11973
}

\section{Abstract}

The VUV storage ring at the National Synchrotron Light Source was used to study the impact of changes in the momentum compaction factors over a large range from positive to negative values. Changes in bunch length and synchrotron tune were measured versus current and RF parameters for these different lattices. By controlling both the first and second-order momentum compaction factors, a lattice was developed in which a pair of alpha buckets was created within the energy aperture of the vacuum chamber and beam was stored simultaneously in both buckets.

\section{INTRODUCTION}

The longitudinal equations of motion for electrons are given as,

$$
\begin{aligned}
& \dot{\phi}=-\omega_{\mathrm{rf}}\left(\alpha_{1}-\alpha_{2} \delta\right) \delta \\
& \dot{\delta}=\frac{\mathrm{eV}_{\mathrm{rf}} \operatorname{Sin}\left(\phi+\phi_{\mathrm{s}}\right)-\mathrm{U}(\delta)}{\mathrm{E}_{0} \mathrm{~T}_{0}}
\end{aligned}
$$

where $\delta \equiv \Delta E / E_{0}$, and $\alpha_{1}, \alpha_{2}$ are the first and secondorder momentum compaction factors respectively. The need to consider higher order terms in the momentum compaction has its origins in the problem of transition crossing in proton machines [1]. In fact graphic illustrations of the so-called alpha buckets can be found in these early references. More recently several groups at electron storage ring facilities have attempted to develop short electron bunches by reducing $\alpha_{1}$ [2\& refs. therein]. Although this approach can yield short bunches at low currents, it has so far failed at high currents due to increases in the energy spread of the stored beam resulting from a lower threshold for the microwave instability [3]. The infrared users of the VUV ring at the NSLS desire shorter bunch lengths for pump-probe experiments. This requirement motivated a study of the possibility of variable $\alpha_{1}$ lattices and their impact on the bunch length for high current beams in this ring. The VUV ring is normally operated as a medium emittance (160 nm) double bend achromatic lattice and a low frequency (53 $\mathrm{MHz}$ ) RF system.

\section{VARIABLE $\alpha$ LATTICES}

The achromatic condition for the VUV ring is controlled by a single quadrupole family (Q3) in the dispersion region between the two-dipole magnets. By increasing the strength of this quadrupole family the dispersion function can be made negative in a portion of each dipole magnet resulting in a reduction in $\alpha_{1}$. In order to maintain injection, the betatron tunes are restored using the remaining two quadrupole families (Q1 \& Q2). Varying the Q3 quadrupole over a range of $40 \%$ allowed the $\alpha_{1}$ to be varied over a wide range, $-\alpha_{10}<\alpha_{1}<2 \alpha_{10}$, where $\alpha_{10}=0.0235$ is the value for the achromatic lattice. The value of $\alpha_{1}$ was determined for each lattice by measuring the synchrotron frequency (excited by a kicker), $\mathrm{f}_{\mathrm{s}} \propto \sqrt{\alpha_{1}}$, for $\mathrm{I} \approx 1 \mathrm{ma}$; the low current was chosen to eliminate the effects of potential well distortion. Figure 1 shows these values for $\left|\alpha_{1}\right|$ as a function of the magnetic field measured in a Q3 magnet.

For $\alpha_{1} \geq \alpha_{10} / 10$ we attempted an independent measurement of $\alpha_{1}$ by using the lattice modeling code LOCO [4]. Since this program required measurements of the beam response matrices, we were able to use it only where sufficient current could be stored. We also measured lattices with $\alpha_{1}=-\alpha_{10}$ where the sextupoles were turned off to store high current, since the head-tail instability was not a problem for negative (natural) chromaticity. The values of $\alpha_{1}$, calculated by MAD using the LOCO determined lattice, are also shown in Figure 1. Although the LOCO calculations yield results close to the values estimated by the synchrotron tune measurements, there remains some significant differences. For the remainder of the studies we adopted the synchrotron tune measurement scheme to determine $\alpha$.

As the magnitude of $\alpha_{1}$ is reduced, we needed to consider the impact of $\alpha_{2}$. For $\alpha_{2} \neq 0$, a stable off energy RF bucket exists at $\delta_{\mathrm{e}}=\alpha_{1} / \alpha_{2}$ in addition to the "normal" one at $\delta_{\mathrm{o}}=0$. As $\alpha_{1}$ decreases two things happen: 1) the energy deviation, $\delta_{\mathrm{e}}$, decreases so that the two buckets approach one another and 2) the height of each bucket increases as $\delta_{\text {rf } 0} \propto \sqrt{\mathrm{V}_{\text {rf }} / \alpha_{1}}$. Collision of the two buckets occurs when $\delta_{\text {rf } 0}=\alpha_{1} / \sqrt{3} \alpha_{2}$. After collision, the energy acceptance of the buckets depends only on $\alpha_{1} / \alpha_{2}$ and the phase acceptance of the buckets is given by

$$
\Delta \phi \approx 2 \frac{1}{\sqrt{3}} \frac{\alpha_{1}}{\alpha_{2}} \sqrt{\frac{2 \pi \mathrm{h} \alpha_{1} \mathrm{E}_{0}}{\mathrm{eV} \mathrm{V}_{\mathrm{rf}} \cos \phi_{\mathrm{s}}}} .
$$

By controlling $\alpha_{2}$ using the SF chromaticity correcting sextupole magnets we were able to store beam in lattices with $\alpha_{1} \approx \alpha_{10} / 200$.

\footnotetext{
* Work supported by U.S. DOE contract DE-AC02-98CH10886.

" Email address: kramer@bnlls1.bnl.gov
} 


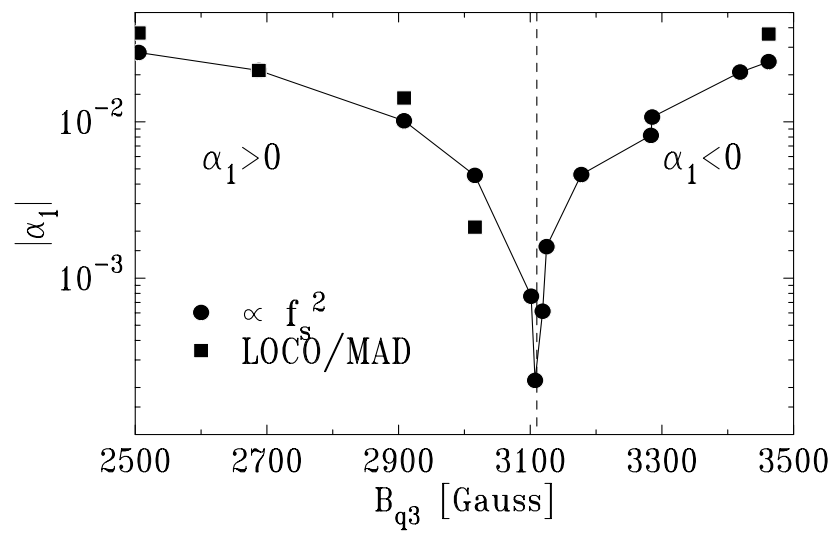

Figure 1: Variation of $\left|\alpha_{1}\right|$ with the magnetic field in the Q3 quadrupole family, Q1 and Q2 are varied to maintain constant tunes.

The values for $\alpha_{2}$ were measured by varying the RF frequency, $\mathrm{f}_{\mathrm{rf}}$, using the relationship [2]

$$
\mathrm{f}_{\mathrm{s}}^{4}=\mathrm{f}_{\mathrm{s} 0}^{4}\left(1+4 \frac{\alpha_{2}}{\alpha_{1}^{2}} \frac{\Delta \mathrm{f}_{\mathrm{rf}}}{\mathrm{f}_{\mathrm{rf}}}\right) .
$$

\section{STORED BEAM IN ALPHA BUCKETS}

On March 16, 1999, we successfully stored electrons in a pair of alpha buckets; either bucket alone or both simultaneously. This was achieved not by reducing $\alpha_{1}$ to the smallest values $\left(\alpha_{1} \approx \alpha_{10} / 10\right)$, but by increasing $\alpha_{2}$ such that the value $\delta_{\mathrm{e}} \leq 1 \%$, a value devised to maintain the off energy bucket inside the vacuum chamber aperture on the off energy closed orbit. To provide adequate phase acceptance, $\mathrm{V}_{\mathrm{rf}}$ was reduced by at least a factor of 3-4. Figure 2 shows the two bunches as measured on a stripline BPM; superimposed is the waveform of the $53 \mathrm{MHz}$ RF cavity field. The time separation for the two alpha bunches is $6.75 \mathrm{~ns}$ or $\Delta \phi=$ $128.5^{\circ}$ of the RF wave. This spacing is $\Delta \phi=\pi-2 \phi_{\mathrm{s}}$ and was tunable by changing $\mathrm{V}_{\mathrm{rf}}$. Note that the separation between "normal" RF buckets in the VUV ring is 18.9 ns. In Figure 3 the 2-D images of the two bunches are visible on a synchrotron radiation monitor. The separation $\Delta \mathrm{x}=3.3 \mathrm{~mm}$ for the left image is proportional to the energy difference of the two bunches with $\delta_{o}$ on the left and $\delta_{\mathrm{e}}$ on the right, the reverse of the scope trace in Figure 2. The dispersion is not well known at this beam port, however using two different BPM measurements of $\Delta \mathrm{x}$ and the calculated dispersion from the LOCO model we determine $\delta_{\mathrm{e}}=\Delta \mathrm{x} / \mathrm{D}_{\mathrm{x}}=0.66 \%$. The $\Delta \mathrm{x}$ separation was tunable by changing $\alpha_{2}$ with the SF sextupole, as shown in the right image of Fig. 3 where $\delta_{\mathrm{e}} \approx 0.4 \%$. It can also be seen that there is a vertical separation of the beam spots, which indicate the presence of vertical dispersion in the ring. To arrive at the alpha bucket lattice that we could inject into we'd greatly increased the strength of SF to increase $\alpha_{2}$. We believe the horizontal chromaticity was pushed far positive while sending the vertical chromaticity negative putting us at odds with the head-tail instability. In normal operation the VUV ring can store roughly 15 ma without any sextupoles (head-tail limit) and this jives with the current limit we could attain in the on energy alpha bucket. At present we were limited to $2 \mathrm{ma}$ in the off energy bucket.

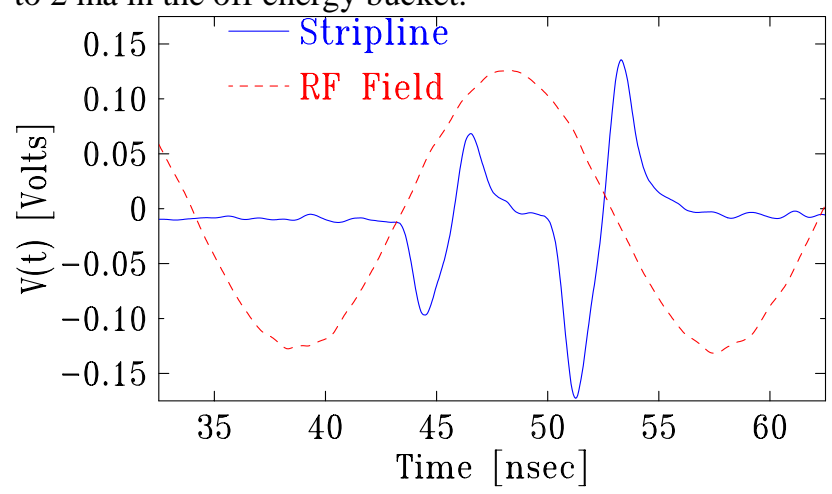

Figure 2: Scope traces of the RF field and beam bunch signals from a stripline BPM showing beam stored in the two alpha buckets separated by $6.75 \mathrm{~ns}$.
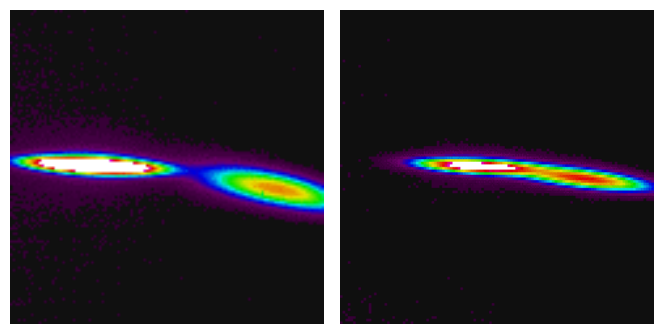

Figure 3: Synchrotron light monitor images showing the two alpha buckets for two values of $\alpha_{2}$. The horizontal bunch spacing, $\Delta x=3.3$ and $2.0 \mathrm{~mm}$ for left and right images respectively, are proportional to $\delta_{\mathrm{e}}$.

\section{BUNCH LENGTH MEASUREMENTS}

The bunch length was first measured with a fast photodiode and a $20 \mathrm{GHz}$ sampling scope. The response of the diode and scope was measured with a 2 psec laser pulse and gave a Gaussian risetime of 28 psec. The measured risetime, $\sigma_{t}$, assuming a Gaussian leading edge to the bunch, was obtained by subtracting in quadrature the delta function response from the measured bunch risetime. A second method used a fast transient digitizer to measure the bunch shape in a single passage of the bunch [5]. This used a beam position monitor, BPM, to obtain a large coupling to the beam. The resulting signal was deconvolved using a measured response function determined at low current by assuming a Gaussian bunch shape as measured by the diode.

Figure 4 shows the measured $\sigma_{\mathrm{t}}$ of the bunch as a function of current. This data shows the reduction in the 
zero current bunch length $\sigma_{\mathrm{t}}(\mathrm{I}=0) \equiv \sigma_{\mathrm{o}}$ as $\alpha_{1}$ was reduced. However, the rate of increase with current is greater for smaller values of $\alpha_{1}$. The values of $\sigma_{t}$ measured for $\alpha_{1}= \pm \alpha_{10}$ agreed closely. The data for $\alpha_{10}$ was fit to a bunch lengthening model used for SPEAR data [6], gave a broad-band impedance of $|\mathrm{Z} / \mathrm{n}|=1.8 \Omega$ and a high frequency cutoff parameter $\mathrm{a}=-0.78(\mathrm{a}=-0.68$ for SPEAR). This predicted a threshold for the microwave instability of about $82 \mathrm{~mA}$. However, extrapolating the microwave lengthening to $\sigma_{\mathrm{o}}$ gave a threshold current value of $\mathrm{I}_{\mathrm{m}} \approx 113 \mathrm{~mA}$. It's clear from Figure 4 that $I_{m}$ decreases as $\alpha_{1}$ decreases.

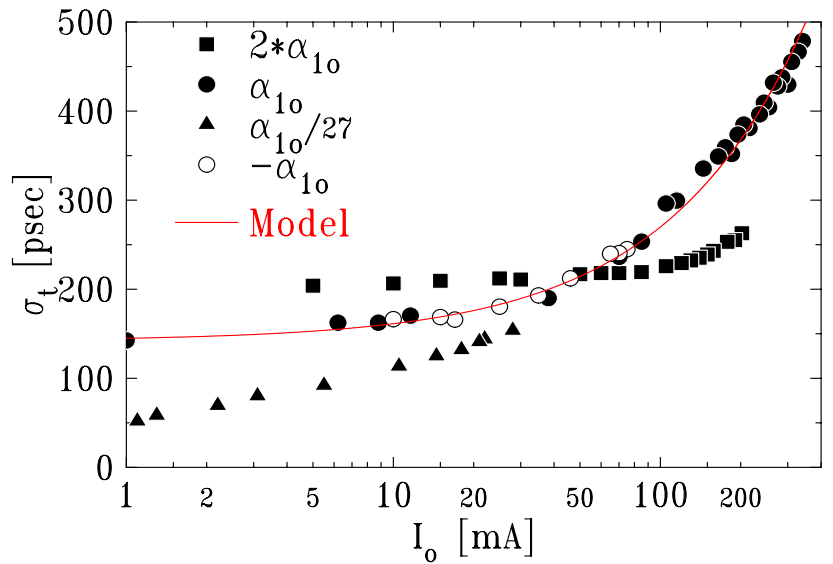

Figure 4: Gaussian risetime measured as a function of bunch current for several values of $\alpha_{1}$.

The measured Gaussian risetime doesn't show the detailed information on the impedance seen by the beam. The reconstructed bunch current distributions using the BPM signal are presented in Figure 5 and show that the FWHM bunch length is larger for $\alpha_{1}>0$ than for the same magnitude of $\alpha_{1}<0$. This appears to be the result of a sharper trailing edge to the bunch distribution rather than from the leading edge, risetime show in Figure 4. This is qualitatively consistent with a resistive impedance plus and inductive term which should contribute to bunch lengthening for $\alpha_{1}>0$ and shortening for $\alpha_{1}<0$ [7].

\section{IR COHERENT SR THRESHOLDS}

Infrared users on the VUV Ring have seen large bursts of far-infrared synchrotron radiation being emitted by the beam. These signals are most prominent above a welldefined current threshold, $I_{c}$ and increase in intensity proportional to the bunch current squared [8]. For the achromatic lattice, $I_{c}$ agrees with $I_{m}$ calculated using the bunch length data. Since this threshold should depend on the bunch length and therefore $\alpha_{1}$, we compare $I_{c}$ with $\mathrm{I}_{\mathrm{m}}$ for the different $\alpha_{1}$ lattices in the following table. For $\alpha_{1}>0, I_{c} \approx I_{m}$, but for $\alpha_{1}<0, I_{c}<I_{m}$. This agrees with the shorter FWHM bunches for $\alpha_{1}<0$.

\begin{tabular}{|l|l|l||l|l|l|}
\hline$\alpha_{1}>0$ & $\mathrm{I}_{\mathrm{c}}[\mathrm{mA}]$ & $\mathrm{I}_{\mathrm{m}}[\mathrm{mA}]$ & $\alpha_{1}<0$ & $\mathrm{I}_{\mathrm{c}}[\mathrm{mA}]$ & $\mathrm{I}_{\mathrm{m}}[\mathrm{mA}]$ \\
\hline 0.030 & 148 & 140 & -.026 & 60 & 127 \\
\hline 0.0235 & 100 & 113 & -.023 & 56 & 113 \\
\hline 0.0109 & 54 & 56 & -.012 & 30 & \\
\hline 0.0049 & 18.5 & 17 & -.005 & 10.5 & \\
\hline 0.0008 & 4.2 & & -.0007 & 2.5 & \\
\hline
\end{tabular}

\section{ACKNOWLEDGEMENTS}

We gratefully acknowledge the assistance of James Safranek at SSRL with his LOCO code. We thank David Robin at LBNL for useful discussions. We also are grateful to G. Ramirez and the NSLS operations staff for assistance during the machine studies.

\section{REFERENCES}

[1] A.A. Kolomenski \& L.L. Sabsovich, p. 112, CERN 56-25 (1956), and N. Vogt-Nilsen, CERN PS/NVN-2 (1958).

[2] D. Robin, et. al., "Experimental Results on Low Alpha Electron Storage Rings", AIP Conf. proceedings 367, p. 150 (1996).

[3] C. Limborg, "A Review of Difficulties in Achieving Short Bunches in Storage Rings", Proc. EPAC-98, p. 151 (1998).

[4] J. Safranek, "Experimental Determination of Storage Ring Optics Using Orbit Response Measurements", Nuc. Instr. and Meth., A388, p.27 (1997).

[5] S.L. Kramer, et. al., "Bunch Current Density Measurements in the VUV Light Source", proceedings of EPAC-94, p. (1995).

[6] P. Wilson, et. al., "Bunch Lengthening and Related Effects in SPEAR II”, IEEE Trans. Nuc. Sci., NS-24, p.1211 (1977).

[7] Karl L.F. Bane, "Bunch Lengthening in the SLC Damping Rings", SLAC-PUB-5177, (1990).

[8] G.L. Carr, et. al., "Investigation of Coherent Emission from the NSLS VUV Ring", this proceedings (1999).

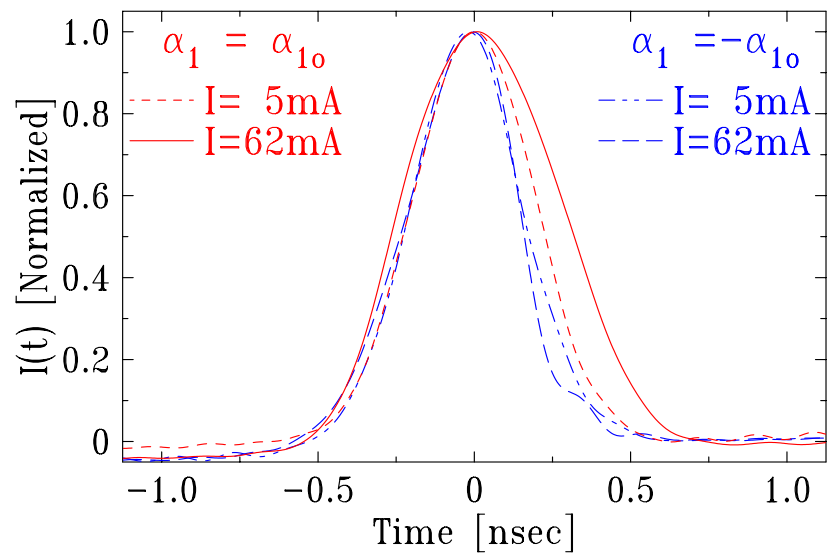

Figure 5: Bunch current distribution measured for two beam currents ( 5 and $62 \mathrm{~mA}$ ) and for $\alpha_{1}= \pm \alpha_{10}$. 